\title{
Assessment of a mathematical model considering the effect of flow rate on in-vitro drug-release from PLGA films
}

\author{
Navideh Abbasnezhad ${ }^{1,2 *}$, Farid Bakir ${ }^{1}$, Stéphane Champmartin ${ }^{1}$, Mohammadali Shirinbayan ${ }^{1,2}$ \\ ${ }^{1}$ Arts et Métiers Institute of Technology, CNAM, LIFSE, HESAM University, F-75013 Paris, France \\ ${ }^{2}$ Arts et Métiers Institute of Technology, CNAM, PIMM, HESAM University, F-75013 Paris, France
}

\begin{abstract}
Drug-eluting stents implanted in blood vessels are subject to various dynamics of blood flow. In this study, we present the evaluation of a mathematical model considering the effect of flow rate, to simulate the kinetic profiles of drug release (Diclofenac Sodium (DS)) from in-vitro from PLGA films. This model solves a set of non-linear equation for modeling simultaneously the burst, diffusion, swelling and erosion involved in the mechanisms of liberation. The release parameters depending on the flow rate are determined using the corresponding mathematical equations. For the evaluation of the proposed model, test data obtained in our laboratory are used. To quantify DS release from drug-carrier PLGA films, we used the flow-through cell apparatus in a closed-loop. Four flow rate values are applied. For each value, the modelsubstance liberation kinetics showed an increase in drug released with the flow rate. The simulated release profiles show good agreement with the experimental results. Therefore, the use of this model could provide a practical tool to assess in-vitro drug release profiles from polymer matrices under continuous flow rate constraint, and could help improve the design of drug eluting stents.
\end{abstract}

\section{Introduction}

Drug release profile plays an important role in the efficacy of the therapy. The design of prolonged and controlled medicine administration systems represents a subject of importance and complex to control. Moreover, a profound understanding of the physical mechanisms contributing to shape the kinetic profile for the drugcarriers is necessary in this regard. However total comprehension of the release mechanisms due to the complexity of delivery system is not easy to accomplished. But achieving this objective will help in the design and optimization of these devices. Mathematical models in this regard, can be helpful to decrease the time and costs of development. However, the validation of the model by experimental tests is among the important steps of the modeling approach. Currently, for predicting drug release profiles, two distinct approaches are taking into account: either by considering the equations of the physical mechanisms involved [1-4] or by considering the kinetics of the release profile [5-8]. Studies showed that the drug release profile may contain different stages. Generally five different phases are known: lag time, burst release, slow release, fast release, extended release. The existence of one of these stages is due to the different release mechanisms involved in the drug delivery system and also to external conditions [8]. To ensure the success of any type of modeling, it is necessary to identify the major influence of many factors affecting the release profile like temperature, initial drug load or flow rate.

In this work we present an approach based on the resolution of a set of non-linear equations modeling the mechanisms of burst-release, diffusion, swelling and erosion. The chosen application concerns the samples of a biodegradable PLGA matrix loaded with $10 \%$ of DS. The experimental data employed take into account several flow rates of the fluid circulating in a pipe simulating the blood flow in stented arteries for in-vitro conditions [9].

\section{Materials and methods}

\subsection{Mathematical modeling}

Depending on the type of delivery system, the timeprofile of drug liberation results from one or more physical mechanisms occurring simultaneously or consecutively. Each of these mechanisms contributes to a part of the global quantity released, with a value varying from $0 \%$ and $100 \%$. The sum of all the proportions is, of course, $100 \%$ of the global quantity released. We therefore propose the following model:

$$
\begin{gathered}
\frac{\boldsymbol{M}_{\boldsymbol{t}}}{\boldsymbol{M}_{\infty}}=\sum_{\boldsymbol{i}=1}^{\boldsymbol{i}=\boldsymbol{N}} \boldsymbol{\mu}_{\boldsymbol{i}} \times \boldsymbol{F}_{\boldsymbol{i}} \\
\sum_{i=1}^{\boldsymbol{i}=\mathrm{N}} \boldsymbol{\mu}_{\boldsymbol{i}}=1, \text { and } 1 \geq \boldsymbol{\mu}_{\boldsymbol{i}} \geq \mathbf{0}
\end{gathered}
$$

$\boldsymbol{M}_{\boldsymbol{t}}$ represents the quantity of drug released at time $t$, $\boldsymbol{M}_{\infty}$ the amount initially loaded, $\mu_{i}$ the contribution of each of the $\mathrm{N}$ mechanisms involved and $\boldsymbol{F}_{\boldsymbol{i}}$ the equation of each of the various mechanisms. Each equation contains a certain number of unknowns depending on the 
type of drug, drug-carrier, conditions of the surrounding medium, etc.

The proposed method comprises solving a set of nonlinear equations modeling the selected mechanisms. The operated method, known as one of the most effective optimization procedures for solving non-linear optimization problems, is the Sequential Quadratic Programming algorithm [10]. Such a problem consists in calculating the factors minimizing a function and simultaneously respecting the imposed constraints. It is a Newtonian algorithm applied to the first order optimality conditions of the system. It finds the solution of the nonlinear problems through linear approximations. The quadratic sub-problem (QP) generated is solved following the steps described in the flowchart below (shown in Fig. 1).

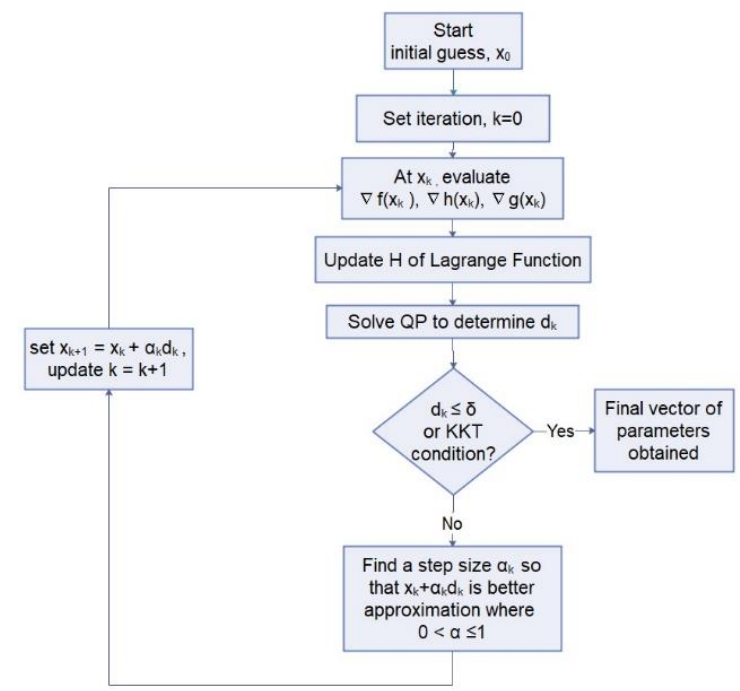

Fig. 1. The iterative flowchart of the SQP method [11]

\subsection{Materials and in-vitro release assays}

In this paper, we presented the release profile at different flow rates, $0,6.5,7.5$ and $15 \mathrm{ml} / \mathrm{s}$, of the chosen drug model, Diclofenac Sodium (DS), from films of biodegradable PLGA samples loaded with $10 \%$ DS. We keep the nomenclature below for the identification of the different cases "PLGA - concentration level of DS - flow rate in $\mathrm{ml} / \mathrm{s}$. For example, PLGA-10\%DS-Q15.

The samples were prepared with the $20 \% \mathrm{w} / \mathrm{v}$ PLGA (50:50) to the solvent (ethyl acetate) with the mixture of the $10 \% \mathrm{w} / \mathrm{w}$ of the DS to the polymer [12]. Initially, a solution of the drug and of the solvent was prepared, next it was agitated with a magnetic stirrer until completely homogenizing the drug in the solvent. Then the weighted PLGA was added to the solvent and finally the solution was agitated again at $700 \mathrm{rpm}$ for about 1 hour. In the next step, the solution was poured into the mold covered with Teflon and was dried at room temperature for 24 hours. Finally, the mold was placed in the vacuum oven at the temperature of $40^{\circ} \mathrm{C}$ for $24 \mathrm{~h}$. Rectangular films have been sliced with the dimensions of $30 \times 5 \times 0.3 \mathrm{~mm}^{3}$.
The experimental results were obtained on a test bench (shown in Fig. 2), which was configured to generate different flow rates of the PBS solution. The flow rate was regulated with the pump and valves on the system, which were controlled via acquisition card connected to LabVIEW. A reservoir with the capacity of 1 Litre is considered. The sample was placed in the chamber, was contacted on one side with the flow medium and the other side with a rigid surface. The tests have been performed at the temperature of $37^{\circ} \mathrm{C}$ and $\mathrm{pH}$ of 7.4 and at each time step $4 \mathrm{ml}$ of the solution was sampled for quantification by UV-VIS [13].

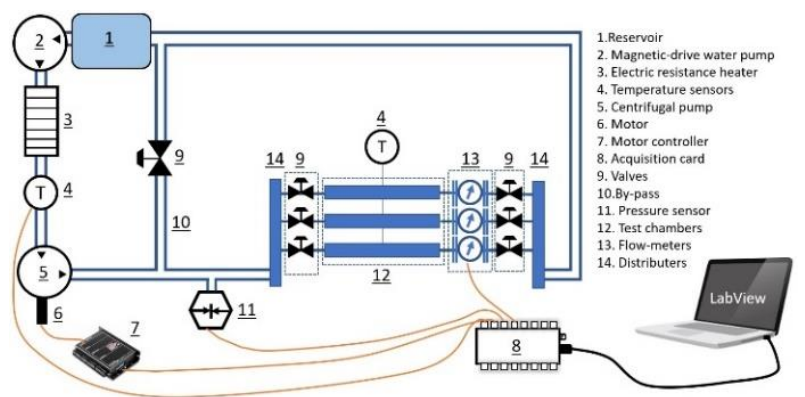

Fig. 1. Experimental drug release from the PLGA matrix with $10 \%$ of drug at different flow rates

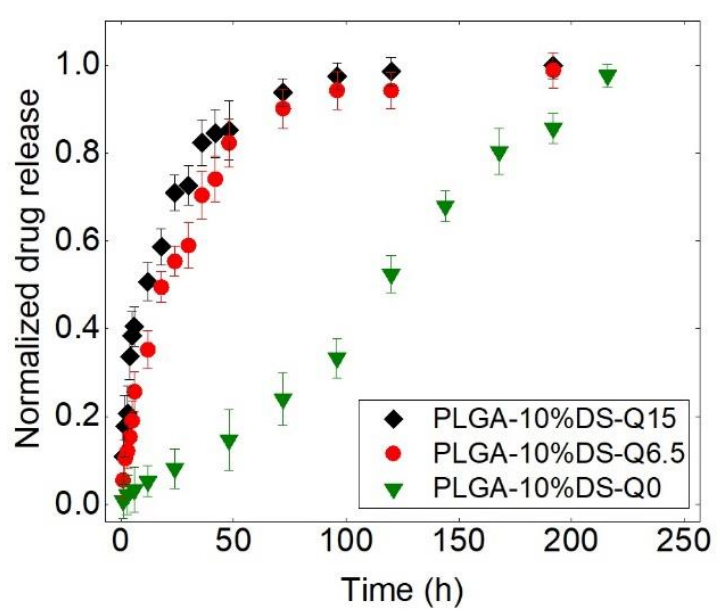

Fig. 2. Experimental drug release from the PLGA matrix with $10 \% \mathrm{DS}$ at the flow rates of $0,6.5 \mathrm{ml} / \mathrm{s}$ and $15 \mathrm{ml} / \mathrm{s}$

From Fig. 3 one can note that the release profile can be divided into three regions. The first region is related to the burst release which is occurring at the very beginning of the release at high speed. It is normally related to the drugs on the surface of the samples. It can be related to the diffusion from the water-filled pores which are connected to the surface of the samples [14]. The second stage which is rather slower is due to the coupling of the diffusion and swelling mechanisms. The effective parameter affecting the diffusion mechanism is the physical state of the polymer. It is well-known that polymers at the rubbery state have a larger diffusion coefficient than at the glassy state. This is related to the glass transition temperature of the polymer during the release time. It can be well described that the $T_{g}$ of the polymer matrix before exposure to the medium is above $37{ }^{\circ} \mathrm{C}$ but during the release time this value is 
decreased by the plasticizing effect of the water molecules [12].

In order to release the drug by diffusion, the drug firstly needs to be dissolved in the polymer and then released from it. This process could decrease the overall release rate [14]. Meanwhile, swelling mechanism is activated by the rapid water absorption. In fact, the hydrophilic polymer rapidly absorbs water and causes the formation a porous structure near the surface, which promotes the drug release. The last stage can be related to the erosion mechanism when the polymer is weak from a mechanical point of view. Here polymer degradation occurs where results in the cleavage of the chains [15]. The other reason would be due to the contact of the surface of the material with the fluid and the friction between them.

Fig. 4 shows the optic observation of the PLGA$10 \% \mathrm{DS}$ after (a) $1 \mathrm{~h}$ and (b) $24 \mathrm{~h}$ of release at the flow rate of $6.5 \mathrm{ml} / \mathrm{s}$. These images show that pores are created at the surface of the samples which accelerate the release rate of the drug.

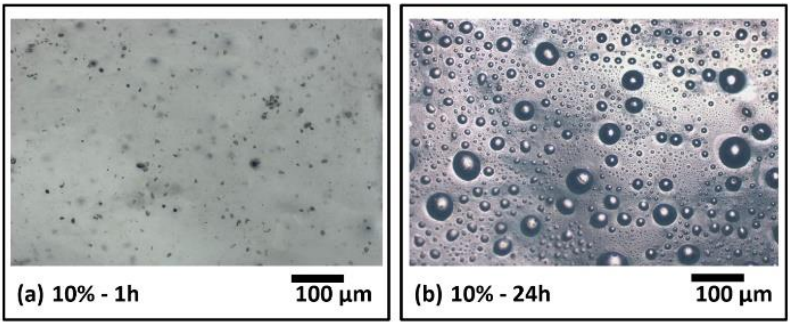

Fig. 3. Comparing the optical observation of the PLGA-10\%DSQ6.5 after (a) $1 \mathrm{~h}$ and (b) $24 \mathrm{~h}$

\subsection{Application of the mathematical model on a loaded PLGA films}

Referring to the type of the drug carrier (PLGA films) and the release conditions, the most common and frequently mentioned mechanisms for drug release are burst-release, diffusion, swelling and erosion [16-20].

$$
\begin{gathered}
\frac{M_{t}}{M_{\infty}}=\mu_{1} \times F_{1}+\mu_{2} \times F_{2}+\mu_{3} \times F_{3}+\mu_{4} \times F_{4} \\
\mu_{1}+\mu_{2}+\mu_{3}+\mu_{4}=1 \\
1 \geq \mu_{1,2,3,4} \geq 0 \\
F_{1}=1-\exp \left(-k_{b} t\right) \\
F_{2}=1-\frac{8}{\pi^{2}} \sum_{n=1}^{\infty} \frac{1}{n^{2}} \exp \left(-\frac{\pi^{2} n^{2} D_{e} t}{h_{1}^{2}}\right) \\
F_{3}=k_{s} t^{\mathrm{m}} \\
F_{4}=1+\exp \left(-2 k_{e} t\right)-2 \exp \left(-k_{e} t\right)
\end{gathered}
$$

where the coefficients $\mu_{1}, \mu_{2}, \mu_{3}, \mu_{4}$ represent respectively the relative contribution of the mechanisms of burst-release, diffusion, swelling and erosion.

The other unknown factors are $\mathrm{k}_{\mathrm{b}}, \mathrm{D}_{\mathrm{e}}, \mathrm{k}_{\mathrm{s}}, \mathrm{k}_{\mathrm{e}}$ and $\mathrm{m}$. Here $\mathrm{m}$ is the specific transport erosion mechanism constant [21], $\mathrm{k}_{\mathrm{b}}$ represents the initial burst kinetics constant, $\mathrm{D}_{\mathrm{e}}$ is an effective diffusivity of solute, $\mathrm{k}_{\mathrm{s}}$ and $\mathrm{k}_{\mathrm{e}}$ are respectively the constants related to the swelling and erosion phenomena. $h_{1}$ is the thickness of the PLGA-film.

\section{Results and discussion}

\subsection{Four-mechanism model (Adjustment BDSE)}

The above definitions can help us to analyse the effect of the flow rate on the kinetics of the different mechanisms. Our model containing the selected above mechanisms (BDSE) was applied to the experimental results of PLGA$10 \% \mathrm{DS}$ with flow rates of $0,6.5$ and $15 \mathrm{ml} / \mathrm{s}$ (Fig. 5 shows the adjustments).

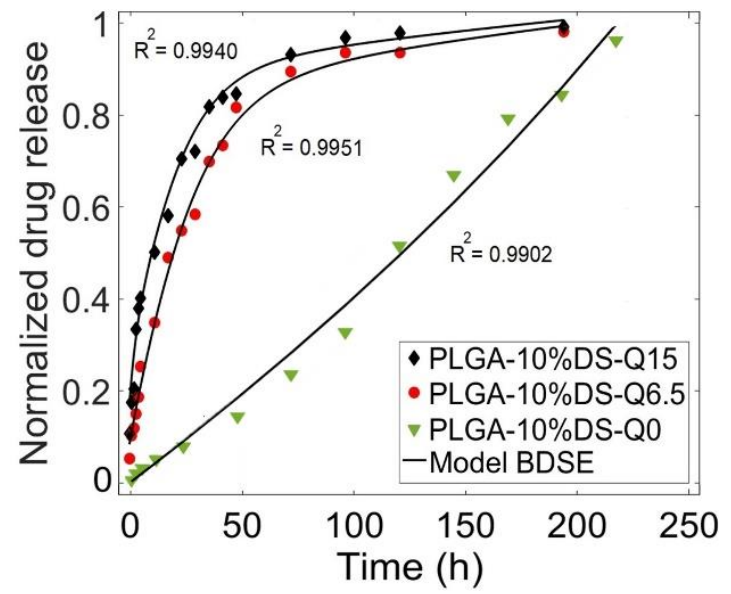

Fig. 5. Experimental drug release from the PLGA matrix with $10 \%$ of drug at the flow rates of $0,6.5$ and $15 \mathrm{ml} / \mathrm{s}$ with the adjustment models (considering burst, diffusion, swelling and erosion mechanisms)

After applying the model (BDSE) on the experimental results, the values related to the contribution of each mechanisms in the drug release and also the kinetics related to them are presented in table 1 .

Table 1. Percentages of each release mechanism and numerical values of the regression parameters at flow rates of $0,6.5$ and 15 $\mathrm{ml} / \mathrm{s}$

\begin{tabular}{cccc}
\hline Mechanism & $\begin{array}{c}\text { PLGA- } \\
10 \% \text { DS-Q0 }\end{array}$ & $\begin{array}{c}\text { PLGA- } \\
10 \% \text { DS-Q6.5 }\end{array}$ & $\begin{array}{c}\text { PLGA- } \\
10 \% \text { DS-Q15 }\end{array}$ \\
\hline Burst (\%) & 0.05 & 18.99 & 21.16 \\
\hline Diffusion (\%) & 0.06 & 8.25 & 9.81 \\
\hline Swelling (\%) & 99.84 & 21.18 & 20.16 \\
\hline Erosion $(\%)$ & 0.05 & 51.58 & 48.87 \\
\hline $\mathrm{k}_{\mathrm{b}}\left(\mathrm{h}^{-1}\right)$ & 0.033 & 0.169 & 0.310 \\
\hline $\mathrm{D}_{\mathrm{e}}\left(\mathrm{m}^{2} / \mathrm{h}\right)\left(\times 10^{-10}\right)$ & 18.00 & 18.00 & 18.00 \\
\hline $\mathrm{k}_{\mathrm{s}}\left(\mathrm{h}^{-1}\right)$ & 0.004 & 0.249 & 0.310 \\
\hline $\mathrm{k}_{\mathrm{e}}\left(\mathrm{h}^{-1}\right)$ & 0.002 & 0.061 & 0.063 \\
\hline $\mathrm{m}$ & 1.00 & 0.72 & 0.70 \\
\hline $\mathrm{R}^{2}$ & 0.9902 & 0.9951 & 0.9940 \\
\hline & & &
\end{tabular}


From the values presented in table 1 it is evident that increasing the flow rate increases the burst release from the drug carriers. It is notable that by changing the state of the flow from static to the continuous, the amount of the burst release is increased which clearly shows that the burst phenomenon is related to convection. It is also clear that the phenomenon of diffusion is enhanced when the flow rate increases. This can be due to the high water uptake. Moreover, in the static state the drug is not able to migrate far away from the sample (thick mass boundary layer and low concentration gradients leading to low diffusive flux), whereas in the continuous state the released drug is immediately transported by the flow (thin mass boundary layer and high concentration gradients leading to high diffusive flux). The mechanism of erosion is also accelerated by the flow rate due to the higher friction between the fluid and the sample, higher shear rate applied to the samples and therefore, rapid weakening of the samples.

When the flow rate is zero, the results show that the mechanisms of burst release, diffusion and erosion are negligible; due to the low shear rate of the flow, low gradient of the concentration and low friction. Therefore swelling is the prevailing mechanism in the static state for this type of sample leading to a slow kinetic and a slow drug release rate.

\subsection{Sensitivity of the model to the choice of mechanisms}

The choice of the mechanisms contributing to the release profile should be carefully chosen. If a particular mechanism is not considered in the model, it will not consistently fit the experimental results. In this regard three examples are given. In the first case, burst release, diffusion and erosion are considered for PLGA-10\%DSQ0 (swelling is neglected). In the second case, burst release, diffusion and swelling mechanisms are considered for PLGA-10\%DS-Q6.5 (erosion is neglected). Finally for the last case, diffusion, swelling and erosion are considered for PLGA-10\%DS-Q15 (burst release is neglected). The results are respectively shown in Fig. 6 (a), (b), (c).

They show that neglecting one mechanism will change the accuracy of the adjustment. Especially when the dominant mechanism of the release is not considered. The values related to the adjustment are shown in table 2 .
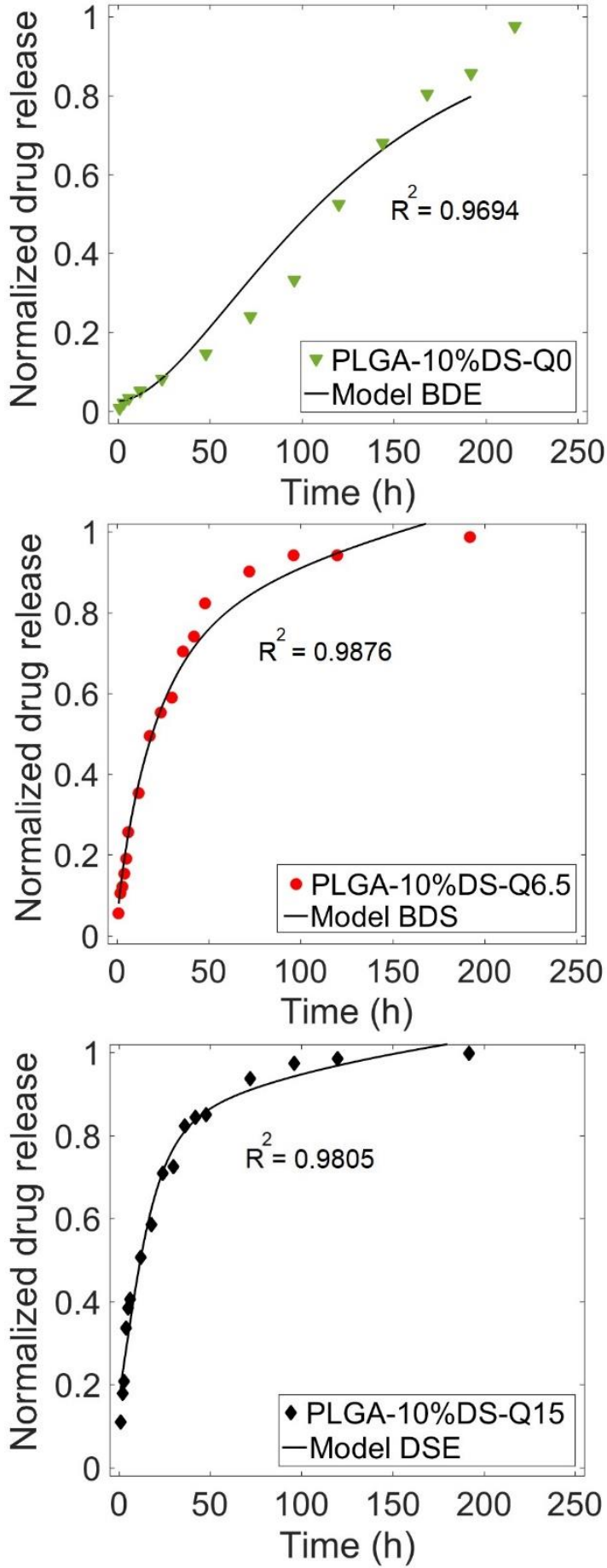

Fig. 6. Experimental drug release from the PLGA-10\%DS-Q0, Q6.5, Q15 with the model adjusted with different mechanisms

Table 2. Values related to the proportion of the contribution of the mechanisms associated in the release and the kinetics related to them

\begin{tabular}{cccc}
\hline Mechanism & PLGA-10\%DS & PLGA-10\%DS & PLGA-10\%DS \\
& $-Q 0$ & $-Q 6.5$ & $-Q 15$ \\
\hline Burst (\%) & 0.01 & 49.99 & - \\
\hline Diffusion (\%) & 2.66 & 0.01 & 5.30 \\
\hline Swelling (\%) & - & 50.00 & 45.45 \\
\hline Erosion (\%) & 97.33 & - & 49.23 \\
\hline $\mathrm{k}_{\mathrm{b}}\left(\mathrm{h}^{-1}\right)$ & 0.005 & 0.014 & - \\
\hline $\mathrm{D}_{\mathrm{e}}\left(\mathrm{m}^{2} / \mathrm{h}\right)\left(\times 10^{-10}\right)$ & 1.296 & 4.752 & 18 \\
\hline $\mathrm{k}_{\mathrm{s}}\left(\mathrm{h}^{-1}\right)$ & - & 11.232 & 115.704 \\
\hline
\end{tabular}




\begin{tabular}{cccc}
\hline $\mathrm{k}_{\mathrm{e}}\left(\mathrm{h}^{-1}\right)$ & 0.011 & - & 0.089 \\
\hline $\mathrm{m}$ & - & 0.43 & 0.26 \\
\hline $\mathrm{R}^{2}$ & 0.9694 & 0.9876 & 0.9805 \\
\hline
\end{tabular}

\subsection{BDSE-Model and its validation}

To find a correlation between the values of constants shown in table 1, figures 7 and 8 were established. In this regard, exponential equations were used to fit the percentage of the contribution of each mechanism $\left(\mu_{1}, \mu_{2}\right.$, $\left.\mu_{3}, \mu_{4}\right)$ and the parameters of each mechanism $\left(\mathrm{k}_{\mathrm{b}}, \mathrm{D}_{\mathrm{e}}, \mathrm{k}_{\mathrm{s}}\right.$, $\left.\mathrm{k}_{\mathrm{e}}, \mathrm{m}\right)$. The correlations between these values give the ability to predict the release for other flow rates.
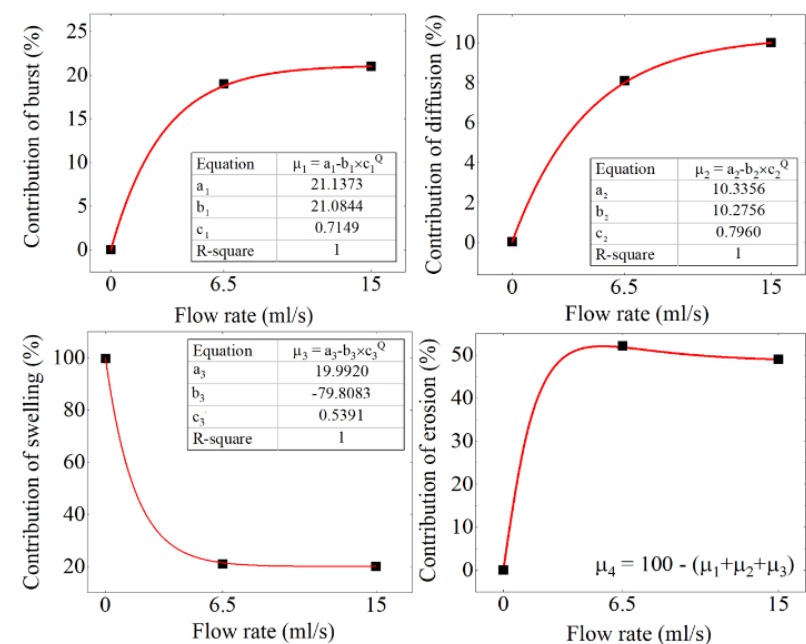

Fig. 7. Exponential fit of the contribution of each mechanisms in drug release vs flow rate
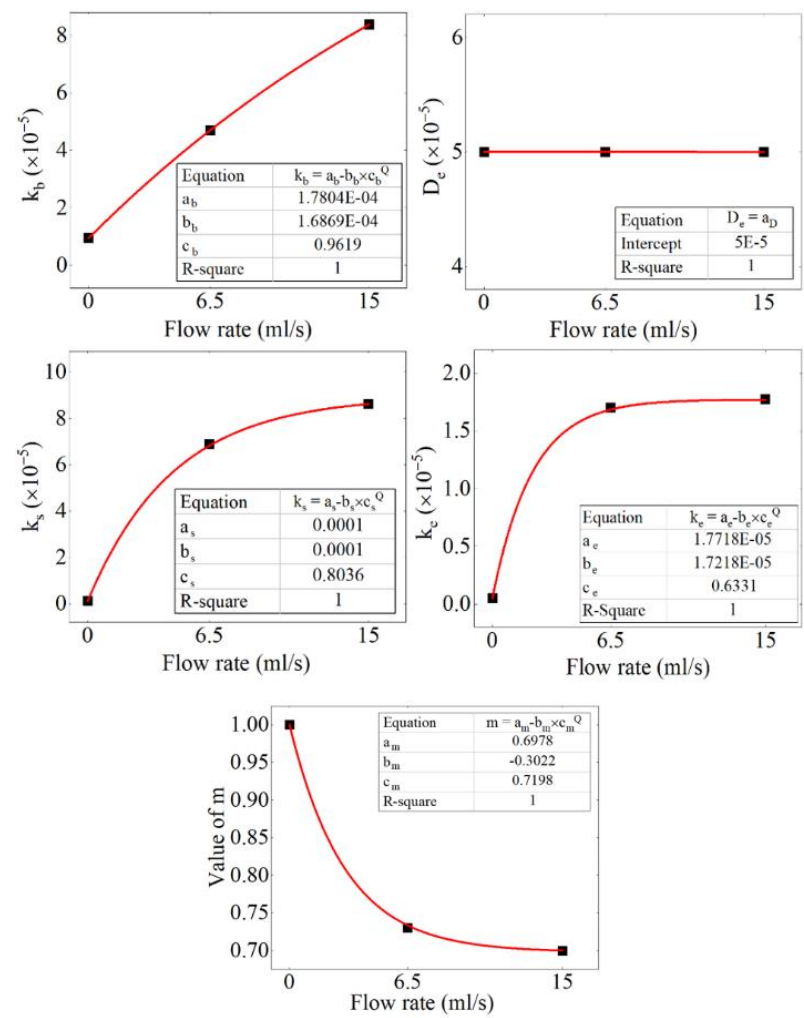

Fig. 8. Exponential fit of the parameters of each release mechanism vs flow rate
Fig. 9 shows the prediction of the drug release profile for the PLGA-10\%DS-Q7.5 by the modeling approach described above and the experimental results obtained. It is notable that the experimental results show a good agreement with the BSDE model obtained by the correlation of the values of constants. Moreover, adjustment profile is also consistent with the model BDSE and the experimental results.

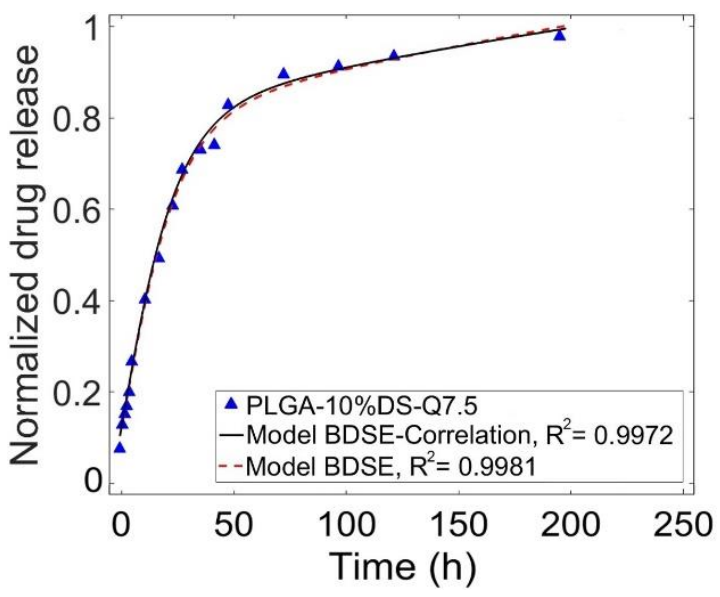

Fig. 4. Comparison between the results of the predicted model and Y-adjustment of BDSE for PLGA-10\%DS-Q7.5 with the experimental results

Table 3 shows the values of constants obtained from modeling and adjustment approach by considering the four mechanisms of burst, diffusion, swelling and erosion for PLGA-10\%DS-Q7.5. The values related to the adjustment and modeling by considering the four mechanisms are in very good accordance.

Table 3. Values related to the proportion of the contribution of the mechanisms associated in the release and the kinetics related to them for PLGA-10\%DS-Q7.5 from the modeling and adjustment approach

\begin{tabular}{ccc}
\hline Mechanism & $\begin{array}{c}\text { PLGA-10\%DS-Q7.5 } \\
\text { Model BDSE-Direct }\end{array}$ & $\begin{array}{c}\text { PLGA-10\%DS-Q7.5 } \\
\text { Model BDSE-Correlation }\end{array}$ \\
\hline Burst (\%) & 19.00 & 20.00 \\
\hline Diffusion (\%) & 8.00 & 8.00 \\
\hline Swelling (\%) & 21.00 & 22.00 \\
\hline Erosion $(\%)$ & 52.00 & 50.00 \\
\hline $\mathrm{k}_{\mathrm{b}}\left(\mathrm{h}^{-1}\right)$ & 0.186 & 0.114 \\
\hline $\mathrm{De}\left(\mathrm{m}^{2} / \mathrm{h}\right)\left(\times 10^{-10}\right)$ & 18.00 & 18.00 \\
\hline $\mathrm{k}_{\mathrm{s}}\left(\mathrm{h}^{-1}\right)$ & 0.260 & 0.198 \\
\hline $\mathrm{k}_{\mathrm{e}}\left(\mathrm{h}^{-1}\right)$ & 0.061 & 0.065 \\
\hline $\mathrm{m}$ & 0.73 & 0.73 \\
\hline
\end{tabular}

\section{Conclusions}

A mathematical model based on the physical mechanisms that can contribute to shaping the drug release profile from carrier-systems is developed. We considered here the four most likely drug release mechanisms which are: burst release, diffusion, swelling and erosion. The influence of the flow rate on the drug-released ratios based on these four mechanisms has been highlighted. 
The unknown variables calculated, forms the necessary data set to elaborate a predictive relationship for this family of carriers. In this regard, the predictive model and the experimental results showed a good correlation. It is notable that the choice of the contributed mechanisms in the drug release should be chosen rigorously.

\section{DECLARATION OF COMPETING INTEREST}

All authors of this manuscript have directly taken part in planning, execution, and/or analysis of this study.

The contents of this manuscript have not been published previously and are not now under consideration for publication elsewhere.

\section{ACKNOWLEDGEMENTS}

The authors wish to acknowledge the financial support of the Fondation Arts et Métiers for this project.

\section{References}

1. Lucero-Acuña, A., Gutiérrez-Valenzuela, C.A., Esquivel, R., Guzmán-Zamudio, R. Mathematical modeling and parametrical analysis of the temperature dependency of control drug release from biodegradable nanoparticles (2019) RSC Advances, 9 (16), pp. 8728-8739. DOI: 10.1039/c9ra00821g

2. Tamani, F., Hamoudi, M.C., Danede, F., Willart, J.-F., Siepmann, F., Siepmann, J. Towards a better understanding of the release mechanisms of caffeine from PLGA microparticles(2020) Journal of Applied Polymer Science, 137 (25), art. no. 48710. DOI: 10.1002/app.48710

3. Fang, Y., Zhang, N., Li, Q., Chen, J., Xiong, S., Pan, W. Characterizing the release mechanism of donepezil-loaded PLGA microspheres in vitro and in vivo(2019) Journal of Drug Delivery Science and Technology, 51, pp. 430-437. DOI: 10.1016/j.jddst.2019.03.029

4. Fernández-Colino, A., Bermudez, J.M., Arias, F.J., Quinteros, D., Gonzo, E. Development of a mechanism and an accurate and simple mathematical model for the description of drug release: Application to a relevant example of acetazolamide-controlled release from a bio-inspired elastin-based hydrogel(2016) Materials Science and Engineering C, 61, pp. 286-292. DOI: 10.1016/j.msec.2015.12.050

5. Abbasnezhad N, Bakir F, Shirinbayan M, Maurel B. New mathematical model based on the kinetic profile for the prediction of multistage drug release from delivery systems. Int J Pharm. 2020;10(2):1-8. ISSN 2249-1848

6. Hammad, R., Khan, I.U., Asghar, S., Khalid, S.H., Irfan, M., Khalid, I., Shah, S.U., Sabir, N., Ali, A., Yousaf, A.M., Hussain, T., Shahzad, Y., Gohar, U.F. Multistage release matrices for potential antiplatelet therapy: Assessing the impact of polymers and SorbCel $M \circledR$ on floating, swelling, and release behavior (2020) Journal of Drug Delivery Science and Technology, 55, art. no. 101387. DOI: 10.1016/j.jddst.2019.101387

7. Tamani, F., Bassand, C., Hamoudi, M.C., Siepmann,
F., Siepmann, J. Mechanistic explanation of the (up to) 3 release phases of PLGA microparticles: Monolithic dispersions studied at lower temperatures (2021) International Journal of Pharmaceutics, 596, art. no. 120220. DOI: 10.1016/j.ijpharm.2021.120220

8. Gasmi, H., Siepmann, F., Hamoudi, M.C., Danede, F., Verin, J., Willart, J.-F., Siepmann, J. Towards a better understanding of the different release phases from PLGA microparticles: Dexamethasone-loaded systems (2016) International Journal of Pharmaceutics, 514 (1), pp. 189-199. DOI: 10.1016/j.ijpharm.2016.08.032

9. Abbasnezhad, N., Shirinbayan, M., Tcharkhtchi, A., Bakir, F. In vitro study of drug release from various loaded polyurethane samples and subjected to different non-pulsed flow rates (2020) Journal of Drug Delivery Science and Technology, 55, art. no. 101500. DOI: $10.1016 /$ j.jddst.2020.101500

10. Boggs, P.T., Tolle, J.W. Sequential Quadratic Programming (1995) Acta Numerica, 4, pp. 1-51. DOI: $10.1017 / \mathrm{S} 0962492900002518$

11. Edgar T.F., Himmelblau D.M., Lasdon L.O.S., Optimization of Chemical Process. McGraw Hill Companies Inc.:New York, NY, USA, 2001

12. N. Abbasnezhad, N. Zirak, M. Shirinbayan, A. Tcharkhtchi, F. Bakir, On the importance of physical and mechanical properties of PLGA films during drug release, Journal of Drug Delivery Science and Technology, 2021, 102446, ISSN 1773-2247, DOI: 10.1016/j.jddst.2021.102446.

13. Abbasnezhad, N., Zirak, N., Shirinbayan, M., Kouidri, S., Salahinejad, E., Tcharkhtchi, A., Bakir, F. Controlled release from polyurethane films: Drug release mechanisms (2021) Journal of Applied Polymer Science, 138 (12), art. no. 50083. DOI: 10.1002/app.50083

14. Siepmann, J., Siepmann, F. Mathematical modeling of drug dissolution (2013) International Journal of Pharmaceutics, 453 (1), pp. 12-24. DOI: 10.1016/j.ijpharm.2013.04.044

15. Göpferich, A. Mechanisms of polymer degradation and erosion (1996) Biomaterials, 17 (2), pp. 103-114. DOI: 10.1016/0142-9612(96)85755-3

16. Huang $\mathrm{X}$, Brazel CS. On the importance and mechanisms of burst release in matrix-controlled drug delivery systems. J Control Release. 2001;73:121-36. DOI: 10.1016/S0168-3659(01)00248-6

17. Yahya I, Atif R, Ahmed L, Eldeen T, Omara A, Eltayeb M. Mathematical Modeling of Diffusion Controlled Drug Release Profiles from Nanoparticles. 2019;4:287-91.

18. Permanadewi I, Kumoro A, Wardhani D, Aryanti N. Modelling of controlled drug release in gastrointestinal tract simulation. J Phys Conf Ser. 2019;1295:12063.

19. Gasmi, H., Willart, J.-F., Danede, F., Hamoudi, M.C., Siepmann, J., Siepmann, F. Importance of PLGA microparticle swelling for the control of prilocaine release (2015) Journal of Drug Delivery Science and Technology, 30, pp. 123-132. DOI: 10.1016/j.jddst.2015.10.009

20. Gasmi, H., Danede, F., Siepmann, J., Siepmann, F. 
Does PLGA microparticle swelling control drug release? New insight based on single particle swelling studies (2015) Journal of Controlled Release, 213, art. no. 7744, pp. 120-127.DOI: 10.1016/j.jconrel.2015.06.039

21. Ritger, P.L., Peppas, N.A. simple equation for description of solute release I. Fickian and non-fickian release from non-swellable devices in the form of slabs, spheres, cylinders or discs (1987) Journal of Controlled Release, 5 (1), pp. 23-36. DOI: 10.1016/0168-3659(87)90034-4 